\title{
Degeneração esponjosa no sistema nervoso central de bezerros da raça Sindhi ${ }^{1}$
}

\author{
Karla M.R.Guedes ${ }^{2}$, Ana L. Schild ${ }^{3}$, Franklin Riet-Correa ${ }^{2 *}$, Severo S. de Barros $^{3}$ e \\ Sara V.D. Simões ${ }^{2}$
}

\begin{abstract}
Guedes K.M.R., Schild A.L. Riet-Correa F., Barros S.S. \& Simões S.V.D. 2006. [Spongious degeneration in the central nervous system in Sindhi calves]. Degeneração esponjosa no sistema nervoso central de bezerros da raça Sindhi. Pesquisa Veterinária Brasileira 26(3):157-160. Centro de Saúde e Tecnologia Rural, Universidade Federal de Campina Grande, Campus de Patos, 58700-000 Patos, Paraíba, Brazil. E-mail franklin.riet@pesquisador.cnpq.br

Spongy degeneration (status spongiosus) includes many histological alterations characterized by vacuoles in the neuropile in different regions of the central nervous system (CNS). The vacuolization can be caused by intramyelinic edema, as in maple syrup urine disease and some toxic diseases, or by astrocytic edema, as in citrullinemia. This paper reports a spongy degeneration in two Sindhi calves, a female and a male. Both calves were offspring from the same bull, and the herd had high degree of inbreeding. The female calf was born normal, but progressive nervous signs were observed since 2 months old. The male calf had progressive nervous signs since it was born. Both calves were euthanized with severe nervous signs when 4 months old. At necropsy the liver was pale in the male calf. Histological alterations were mild to accentuated vacuolization, mainly in the deep cerebral cortex, cerebellar white matter, grey matter of the spinal cord, internal capsule, and brain stem. Fatty degeneration was observed in the liver of both animals. On electron microscopy it was found that the status spongiosus was caused by astrocytic edema. It is suggested that the disease is caused by a metabolic hereditary error.
\end{abstract}

INDEX TERMS: Status spongiosus, astrocytic edema, congenital diseases, cattle.

RESUMO.- Degeneração esponjosa (status spongiosus) agrupa várias alterações histológicas caracterizadas pela formação de vacúolos no neurópilo em diferentes regiões do sistema nervoso central (SNC). Essa vacuolização pode ser por edema intramielínico, como na doença da urina com odor de xarope de bordo (maple syrup urine disease) e algumas doenças tóxicas, ou por edema de astrócitos, como na citrulinemia. Este trabalho descreve degeneração esponjosa do SNC em dois bezerros, um macho e uma fêmea, da raça Sindhi. Ambos são filhos de um mesmo touro, de um rebanho que apresentava alto grau de consangüinidade. Uma fêmea nasceu normal e aos 2 meses apresentou sinais nervosos progressivos. Um macho apresen-

\footnotetext{
1 Recebido em 24 de março de 2006.

Aceito para publicação em 11 de abril de 2006 .

2 Centro de Saúde e Tecnologia Rural, Universidade Federal de Campina Grande, Campus de Patos, 58700-000 Patos, PB, Brazil. E-mail: franklin.riet@pesquisador.cnpq.br

3 Laboratório Regional de Diagnóstico, Faculdade de Veterinária, Universidade Federal de Pelotas, Campus Universitário s/n, Pelotas, RS 96010900 .
}

tou sinais nervosos progressivos desde o nascimento. Os dois foram eutanasiados aos 4 meses de idade com acentuadas alterações do sistema nervoso central. Na necropsia o fígado de bezerro macho estava pálido. Histologicamente havia, em ambos os bezerros, discreta a acentuada vacuolização difusa do SNC, sendo mais acentuada nas camadas profundas do córtex cerebral, cápsula interna, substância branca da medula cerebelar, tronco encefálico e substância cinzenta da medula. No fígado dos dois animais havia degeneração gordurosa nos hepatócitos da região centrolobular. Na microscopia eletrônica observou-se que o status spongiosus era devido a edema astrocitário. Sugere-se que a doença é causada por um erro metabólico hereditário, diferente da doença da urina com odor de xarope de bordo.

TERMOS DE INDEXAÇÃO: Status spongiosus, edema de astrócitos, doenças congênitas, bovinos.

\section{INTRODUÇÃO}

Degeneração esponjosa ou status spongiosus são termos utilizados para agrupar lesões do sistema nervoso central (SNC), em geral letais, caracterizadas por vacuolização do neurópilo, que 
acometem animais e o homem. As lesões são geralmente encontradas na substância branca, mas também há comprometimento da substância cinzenta, podendo ser causada por edema intramielínico ou por edema intracelular nos astrócitos e/ou nos oligodentrócitos, o que somente pode ser confirmado mediante a microscopia eletrônica (Summers et al. 1995). Em bovinos, status spongiosus por edema intramielínico tem sido comprovado nas intoxicações pelas plantas Stypandra imbricata (Huxtable et al. 1980), Helichrysum argyrosphaerum (Van der Lugt et al. 1996), Ateleia glazioviana (Raffi 2004) e Tetrapterys spp (Riet-Correa et al. 2005), na intoxicação por closantel (Borges et al. 1999), na encefalopatia hepática (Summers et al.1995) e na neuromicotoxicose causada pelo fungo Diplodia maydis (Kellerman et al. 1991). Ocorre, também, em algumas doenças hereditárias, das quais a mais conhecida é a doença da urina com odor de xarope de bordo (maple syrup urine disease-MSUD), causada por um erro inato do metabolismo. Esta enfermidade se caracteriza pelo acúmulo dos aminoácidos de cadeia ramificada leucina, isoleucina e valina, causando edema intramielínico (Harper et al. 1986, Baird et al. 1987). Outra doença hereditária dos bovinos, que apresenta vacuolização do neurópilo, é a citrulinemia, que ocorre por acumulação de citrulina nos fluídos corporais devido a uma falha na síntese de argininosuccinato por deficiência de argininosuccinato sintetase (Healy et al. 1990). Histologicamente há um aumento no espaço perineural e perivascular, além de edema intracitoplasmático dos astrócitos (Healy et al. 1990). Na intoxicação pelo antihelmíntico rafoxanida observa-se status spongiosus tanto na substância branca quanto na cinzenta (Prozesky et al. 1977), provavelmente devido edema intracelular (Schröder 1982). Vacuolização do neurópilo observa-se, também, nas encefalopatias espongiformes causadas por príon, que acometem diversas espécies. Nestas doenças, que também apresentam vacuolização neuronal, a vacuolização do neurópilo ocorre pela presença de vacúolos nos axônios e dendritos (Miyashita et al. 2004). O objetivo desse trabalho é descrever os achados epidemiológicos, clínicos e patológicos de uma doença caracterizada por status spongiosus no SNC em bezerros da raça Sindhi no semiárido paraibano.

\section{MATERIAL E MÉTODOS}

A doença foi estudada em dois bezerros da raça Sindhi que foram enviados ao Hospital Veterinário (HV) do Centro de Saúde e Tecnologia Rural (CSTR) da Universidade Federal de Campina Grande (UFCG) com sinais neurológicos. Após identificação dos animais obteve-se o histórico dos casos junto aos tratadores dos animais e realizou-se o exame físico geral dos bezerros e exame específico do sistema nervoso. Após a constatação do agravamento dos sinais clínicos, ambos os animais foram eutanasiados e necropsiados. Para estudo histológico das lesões foram colhidos fragmentos dos órgãos das cavidades torácica e abdominal, músculos esqueléticos, nervo ciático e tireóide, fixados em formol tamponado a $10 \%$, incluídos em parafina, cortados com $6 \mu \mathrm{m}$ de espessura e corados por hematoxila-eosina. O sistema nervoso central foi fixado, também, em formol a $10 \%$ e seccionado transversalmente no córtex (frontal, parietal, temporal e occipital), núcleos da base, tálamo, hipocampo, mesencéfalo na altura dos colículos rostrais, ponte, cerebelo, óbex e medula (cervical, torácica e lombar). Fragmentos de fígado foram cortados em criostato para realização de coloração com Sudan Black para visualização de gordura.
A classificação da intensidade da vacuolização nos cortes histológicos foi feita segundo o modelo utilizado por Harper et al. (1986). Para estudo ultra-estrutural, fragmentos de córtex cerebral, cápsula interna e fígado de um dos bovinos foram fixados em solução de glutaraldeído a $2 \%$ e paraformaldeído a $2 \%$, em tampão cacodilato de sódio, desidratados em uma série crescente de etanóis e incluídos em Epon 812. Os cortes semi-finos foram corados por azul de metileno. Em áreas selecionadas dos blocos foram feitos cortes ultrafinos contrastados por acetato de uranila e citrato de chumbo e observados em microscópio eletrônico de transmissão.

Amostra de urina de um dos animais afetados foi enviada para um laboratório particular (CTN Diagnósticos, Porto Alegre, Rio Grande do Sul) para análise de erros inatos no metabolismo (aminoacidopatias) por Espectrometria de Massa em Tandem.

\section{RESULTADOS}

\section{Histórico e sinais clínicos}

A enfermidade afetou dois animais da raça Sindhi, uma fêmea (Bezerro A) e um macho (Bezerro B), que pertenciam a um rebanho com alto grau de consangüinidade, e eram filhos de um mesmo touro resultante de cruzamento consangüíneo (Fig.1). Os animais eram provenientes de rebanho com manejo semiextensivo, com os bezerros sendo desmamados com aproximadamente sete meses de idade. $O$ rebanho era alimentado durante o período de seca com capim Andrequicé (Leessia hexandra Sw.) picado, administrado no cocho uma vez por dia e, posteriormente, solto em campo nativo característico do semi-árido nordestino.

$\mathrm{O}$ bezerro A nasceu aparentemente normal e mamava sem dificuldade. Aproximadamente aos 2 meses começou a apresentar cansaço, apatia e congestão das mucosas. Neste momento foi tratado com florfenicol apresentando melhora aparente. Esse animal era filho de uma vaca que já parira uma cria com as mesmas alterações e morrera. Aos 3 meses foi levado ao HV, onde ficou internado por 1 mês, apresentando sinais neurológicos progressivos, caracterizados por apatia, depressão, incoordenação motora, andar arrastando as pinças, torção lateral da cabeça, anorexia e salivação (Fig.2).

$O$ bezerro $B$ apresentou comportamento apático desde $o$ nascimento, porém mamava e acompanhava a mãe. Aos 4 meses

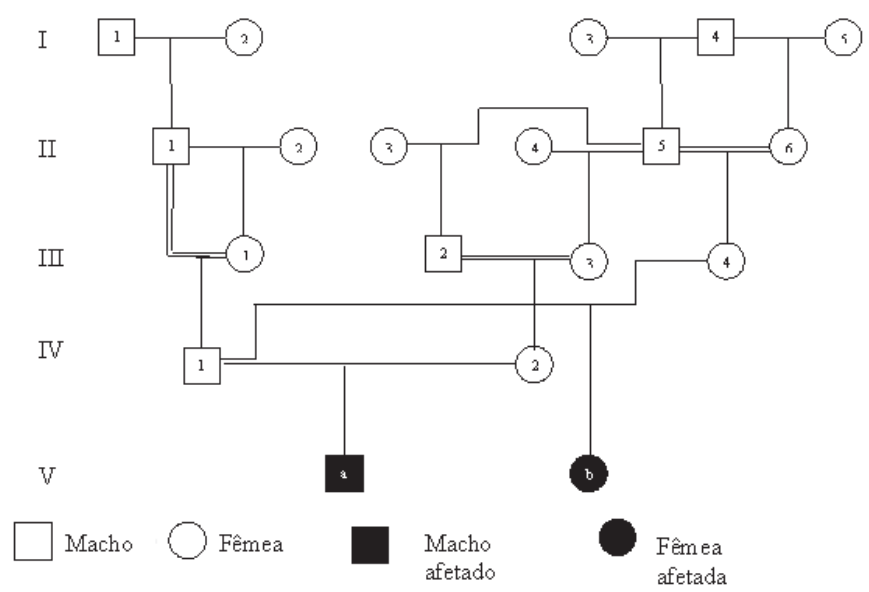

Fig.1. Representação esquemática da árvore genealógica dos animais doentes. 


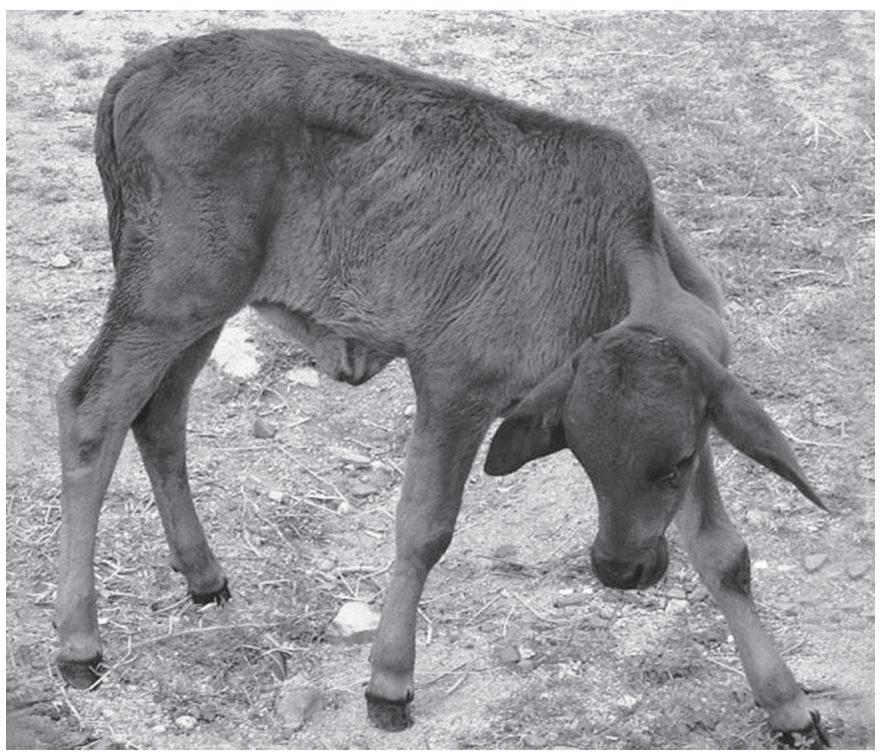

Fig.2. Bezerro A apresentando ataxia, depressão e torção lateral da cabeça.

foi encontrado em decúbito e enviado ao HV. Durante exame físico observaram-se os vasos episclerais ingurgitados e mucosas congestas. Frequientemente, $o$ animal ficava em decúbito lateral levantando-se apenas ao ser estimulado, porém não conseguia manter a estabilidade e voltava ao decúbito lateral ou esternal. Apresentava paresia do membro anterior esquerdo, diminuição do reflexo da ameaça, diminuição da sensibilidade da face, ausência dos reflexos de deglutição e flexão e fasciculação da musculatura do pescoço e membros.

Fig.3. Acentuada vacuolização da substância branca medular no cerebelo (Bezerro A). HE, obj.20.

Fig.4. Fígado com acentuada vacuolização dos hepatócitos da área centrolobular (Bezerro B). HE, obj.10.

Fig.5. Astrócito do córtex cerebral apresentando edema do citoplasma com dilatação das cisternas do retículo endoplasmático (setas). $\mathrm{N}=$ núcleo, $\mathrm{C}=$ citoplasma. Barra $=1 \mu \mathrm{m}$.

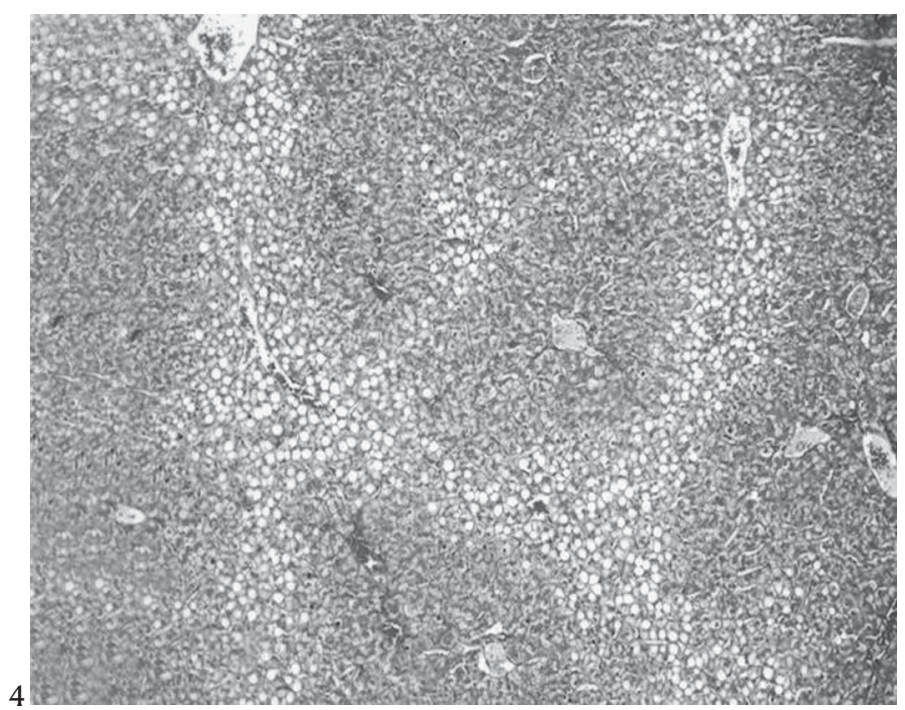

\section{Análise bioquímica}

A análise bioquímica da urina revelou apenas aumento significativo de ácidos láctico, ácido 3-hidróxibutírico e glicose, estando os aminoácidos em quantidades normais.

\section{Patologia macroscópica e histológica}

No Bezerro A não foram observadas lesões macroscópicas de significado patológico e no Bezerro B o fígado estava pálido.

Nos dois bezerros a principal lesão histológica observada foi a vacuolização em diversas áreas do sistema nervoso central, que variou de discreta a acentuada (Fig.3). No telencéfalo a vacuolização se deu principalmente nas camadas profundas do córtex frontal e cápsula interna do Bezerro A. No diencéfalo dos dois animais o corte realizado na altura do tálamo mostrou vacuolização acentuada dos corpos geniculatos lateral e medial e do pulvinar. No mesencéfalo, na altura dos colículos rostral e caudal, observou-se vacuolização acentuada do estrato gríseo intermédio e do fascículo tegmentar. Nos cortes realizados no encéfalo posterior, que engloba cerebelo, ponte, assoalho do quarto ventrículo e óbex, lesões acentuadas foram observadas na medula cerebelar, lemnisco medial, fibra arcuatae interna e núcleo am-

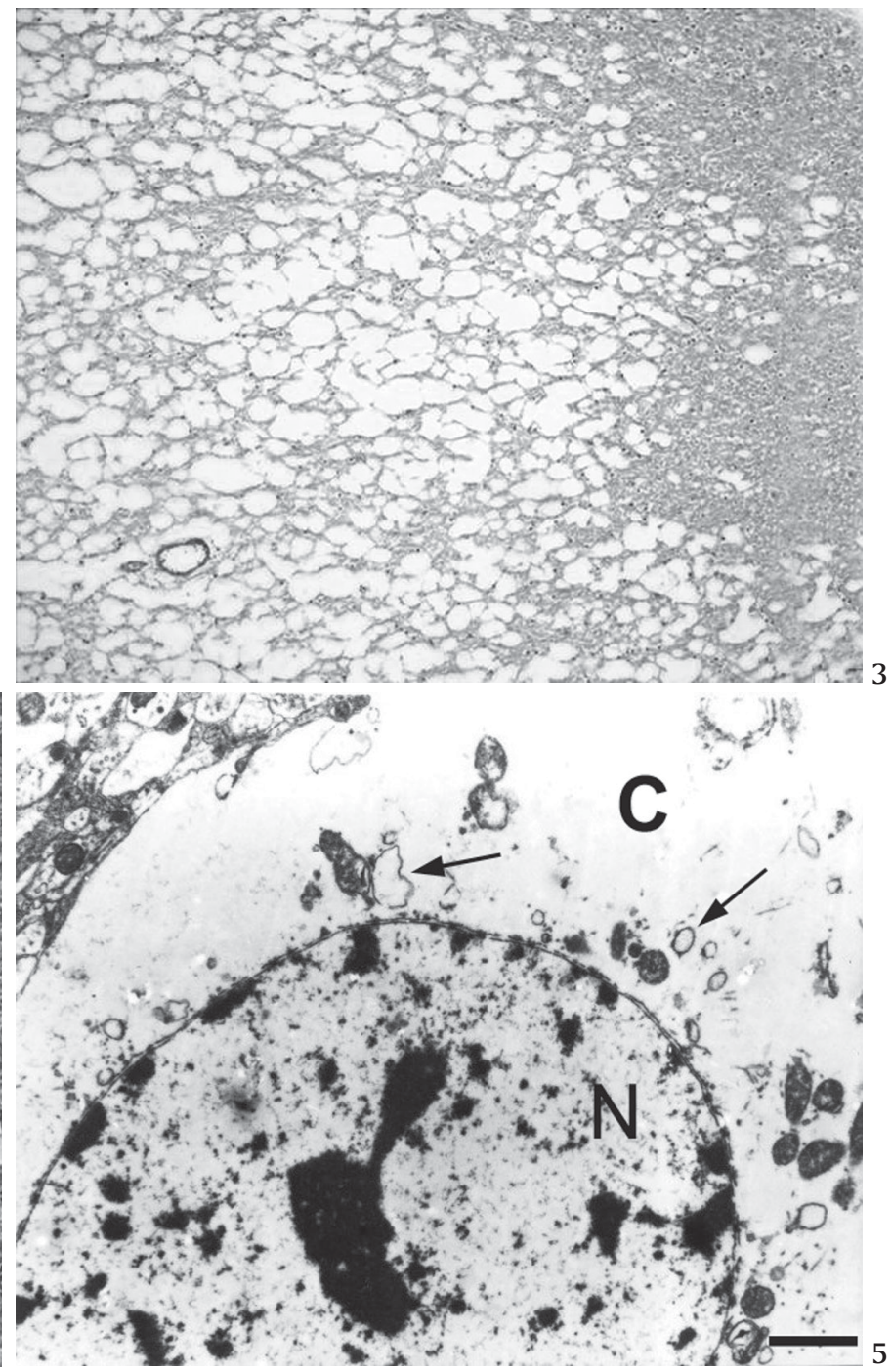


bíguos. Na medula espinhal, os cortes realizados nas três porções (cervical, torácica e lombar) apresentaram vacuolização moderada da substância cinzenta. Alguns neurônios nas áreas afetadas estavam comprimidos, devido, aparentemente, à pressão exercida pelos vacúolos adjacentes. No fígado dos dois animais foi encontrada vacuolização acentuada dos hepatócitos, principalmente da região centrolobular (Fig.4). Na coloração por Sudan Black e na microscopia eletrônica foi constatada degeneração gordurosa nos hepatócitos. Nos demais órgãos não foram observadas alterações de significado patológico.

\section{Microscopia eletrônica}

Ultraestruturalmente observou-se que os vacúolos eram devidos a edema astrocitário. $O$ citoplasma dos astrócitos estava tumefeito exibindo acentuado edema (Fig.5), tanto no seu trajeto pelo neurópilo como também junto aos neurônios e ao redor dos vasos. As cisternas do retículo endoplasmático estavam dilatadas (Fig.5).

\section{DISCUSSÃO}

A doença estudada neste trabalho em bezerros da raça Sindhi caracteriza-se por degeneração esponjosa (status spongiosus) do SNC causada por edema dos astrócitos, sem comprometimento da mielina ou dos neurônios. Trata-se, portanto, de uma enfermidade diferente das intoxicações por Stypandra imbricata (Huxtable et al. 1980), Helichrysum argyrosphaerum (Van der Lugt et al. 1996), Ateleia glazioviana (Raffi 2004), Tetrapterys spp (RietCorrea et al. 2005), Diplodia maydis (Kellerman et al. 1991) e closantel (Borges et al. 1999), da encefalopatia hepática (Summers et al.1995) e da MSUD (Harper et al. 1986, Baird et al. 1987), que são causadas por edema intramielínico.

Edema astrocitário no córtex cerebral é descrito na citrulinemia bovina (Healy et al. 1990). No entanto, a doença estudada neste trabalho é diferente em vários aspectos da citrulinemia. Os sinais clínicos da citrulinemia são observados, no máximo, 3 dias após o nascimento e o curso clínico é de 1-6 dias (Healy et al. 1990), enquanto que na doença do gado Sindhi, tanto o bezerro que nasceu com sinais, quanto o que apresentou sinais aos 2 meses sobreviveram até os 4 meses, quando foram eutanasiados. Na citrulinemia as lesões são encontradas principalmente nas camadas profundas do córtex cerebral (Healy et al. 1990), enquanto que no gado Sindhi observam-se lesões difusas, incluindo tronco encefálico, cerebelo e medula espinhal. Por outro lado o exame da urina foi negativo para aminoacidopatias.

A consangüinidade observada no rebanho e o estudo genealógico sugerem que a doença é de caráter hereditário, transmitida por um gene recessivo autossômico, já que acometeu machos e fêmeas, filhos de pais aparentemente normais e que já tiveram outras crias normais. A MSUD (Dennis et al. 1999) e a citrulinemia bovina (Dennis et al. 1989) são também alterações provocadas por genes autossômicos recessivos, sendo que MSUD afeta a raça Hereford (Harper et al. 1986/1989, Baird et al. 1987) e a citrulinemia animais das raças Holandês preto e branco (Dennis et al. 1989, Healy et al. 1990) e Holandês vermelho e branco (Radostits et al. 1999).
Em conclusão, os sinais neurológicos observados na doença estudada em bezerros da raça Sindhi no estado da Paraíba são causados por status spongiosus do SNC, sendo provavelmente de origem hereditária. Estudos bioquímicos deverão ser realizados em novos casos da enfermidade do gado Sindhi para determinar se a mesma é consequiência de um erro metabólico diferente da MSUD e da citrulinemia.

\section{REFERÊNCIAS}

Baird J.D., Wojcinski Z.W., Wise A.P. \& Godkin M.Ann. 1987. Maple syrup urine disease in five hereford calves in Ontario. Can. Vet. J. 28(8):505511.

Borges A.S., Mendes L.C.N., Andrade, A.L., Machado G.F. \& Peiro J.R. 1999. Optic neuropathy in sheep associated with overdosage of closantel. Vet. Human Toxicol. 41(6):378-380.

Dennis J.A. \& Healy P.J. 1999. Definition of the mutation responsible for maple syrup urine disease in poll shorthorns and genotyping poll shorthorns and poll herefords for maple syrup urine disease alleles. Res. Vet. Sci. 67:1-6.

Dennis J.A. Healy P.J., Beaudet A.L. \& O’Brien W.E. 1989. Molecular definition of bovine arginosuccinato synthetase deficiency. Proc Natl Acad. Sci. 86:7947-7951.

Harper P.A.W., Dennis J.A. Healy P.J. \& Brown G.K. 1989. Maple syrup urine disease: a clinical, pathological and biochemical study. Aust. Vet. J. 66(2):46-49.

Harper P.A.W., Healy P.J. \& Dennis J.A. 1986. Maple syrup urine disease as a cause of spongiform encephalopathy in calves. Vet. Rec. 119:62-65.

Healy P.J., Harper P.A.W. \& Dennis J.A. 1990. Bovine citullinaemia: a clinical, pathological, biochemical and genetic study. Aust. Vet. J. 67(6):255258.

Huxtable C.R., Dorling P.R. \& Slatter D.H. 1980. Myelin oedema, optic neuropathy and retinopathy in experimental Stypandra imbricata toxicosis, Neuropathol. Appl. Neurobiol. 6:221-232.

Kellerman T.S., Prozesky L., Schultz R. Anitra, Rabie C.J., Van Ark H., Maartens B.P. \& Lübben A. 1991. Perinatal mortality in lambs of ewes exposed to cutures of Diplodia maydis (=Sternorcapella maydis) during gestation. Onderstepoort J. Vet. Res. 58:297-308.

Miyashita M., Stierstorfer B. \& Schmahl W. 2004. Neuropathological findings in brains of Bavarian cattle clinically suspected of bovine spongiform encephalopathy. J. Vet. Med. 51:209-215.

Prozesky L. \& Pienaar J.G. 1977. Amaurosis in sheep resulting from treatment with rafoxanide. Onderstepoort J. Vet. Res. 44:257-260.

Radostits O.M., Gay C.C., Blood D.C. \& Hinchcliff K.W. 1999. Veterinary Medicine. A textbook of the diseases of cattle, sheep, pigs, goats and horses. 9th ed. W.B. Saunders, London.

Raffi M.B. 2004. Intoxicação experimental por Ateleia glazioviana em ovinos: patogênese e bases morfológicas da falha reprodutiva, da insuficiência cardíaca e dos distúrbios neurológicos. Tese de Doutorado, Curso de Pós-Graduação em Medicina Veterinária, Área de Patologia Veterinária, Universidade Federal de Santa Maria. 80p

Riet-Correa G., Terra F.F., Schild A.L. Riet-Correa F. \& Barros S.S. 2005. Intoxicação experimental por Tetrapterys multiglandulosa (Malpighiaceae) em ovinos. Pesq. Vet. Bras. 25(2):91-96.

Schröder J. 1982. The safety of injectable rafoxanide in cattle. J. South African Vet. Assoc. 53(1):29-31.

Summers B.A., Cummings J.F. \& Lahunta A. 1995. Veterinary Neuropathology. Mosby-Year Book, St Louis, Missouri.

Van der Lugt J.J., Olivier J. \& Jordaan P. 1996. Status spongiosus, optic neuropathy, and retinal degeneration in Helichrysum argyrosphaerum poisoning in sheep and goat. Vet. Pathol. 33:495-502. 